\title{
A mechanistic model of photoadaptation in microalgae
}

\author{
Richard J. Geider ${ }^{1}{ }^{\bullet}$ \& Trevor Platt ${ }^{2}$ \\ ${ }^{1}$ Department of Biological Sciences, The University of Dundee, Dundee DD $14 \mathrm{HN}$, Scotland, United Kingdom \\ ${ }^{2}$ Marine Ecology Laboratory, Bedford Institute of Oceanography, P. O. B. 1006, Dartmouth, Nova Scotia B2Y 4A2, Canada
}

\begin{abstract}
A mechanistic model is presented that describes changes in the chlorophyll a:carbon ratio of microalgae growing in fluctuating light. A consideration of the mass budgets for cell carbon and chlorophyll during nutrient-sufficient microalgal growth illustrates that imbalances between rates of synthesis and degradation of the macromolecular components of a microalgal cell will result in changes in its biochemical composition. The dynamic equations describing the rate of response of the chl:C and $\mathrm{C}: \mathrm{chl}$ ratios to a change in light level are shown to depend on the magnitude of the change. In particular, the cases of light-limited and light-saturated growth rate are considered. A previously described empirical model of photoadaptation kinetics is shown to be a special case of the new analytical model. In general, we conclude that for a photoadaptive variable defined as the ratio of 2 biochemical constituents of a cell where the denominator is the concentration of the catalyst that is rate-limiting for photosynthesis, then the new, mechanistic model simplifies to a first order differential equation describing the photoadaptation kinetics of the ratio.
\end{abstract}

\section{INTRODUCTION}

The dependence of the biochemical composition of microalgae on photon flux density (PFD) (Cook 1963, Parrot \& Slater 1980, Foy \& Gibson 1982) is one manifestation of physiological plasticity in microalgae (Myers 1970). Any observable property of a microalgal cell that depends on the PFD under which the microalga was grown can be considered as a photoadaptive variable. Examples include cell pigment content, enzyme activities, respiration rate and the parameters of the photosynthesis-light (PI) curve (Falkowski 1980, Richardson et al. 1983).

Several models have been developed to describe the physiological adaptation of phytoplankton to PFD for cells in balanced growth (Shuter 1979, Bannister 1979, Kiefer \& Mitchell 1983). Their predictions are in good agreement with experimental observations of the C:chla ratio and the parameters of the PI curve (Laws et al. 1983, 1985). Although derived independently, all 3 models (Shuter 1979, Bannister 1979, Kiefer \& Mitchell 1983) have closely related formulations (Laws et al. 1983, 1985) and can be shown to be based on the

\footnotetext{
- Current address: Department of Plant Biology, The University of Birmingham, P. O. Box 363, Birmingham B15 2TT, England, U. K.
}

application of the principles of energy and mass conservation (Geider 1984). They are all, however, static descriptions of microalgal physiology and are limited in that they cannot be used to investigate the time course of changes in chemical composition that occur in response to changes in PFD.

The importance of photoadaptation as part of the physiological response of microalgae to a fluctuating light regime in nature has been the subject of recent investigations (Jones 1978, Rivkin et al. 1982a, Falkowski 1983, Smith et al. 1983, Lewis et al. 1984a). Adaptation kinetics have been modelled empirically using a first order differential equation (Falkowski \& Wirick 1981, Rivkin et al. 1982a, Liou \& Eybergen 1982, Lewis et al. 1984a, b).

$$
\mathrm{dR}(\mathrm{t}) / \mathrm{dt}=\mathrm{k}\left[\mathrm{R}_{\mathrm{w}}(\mathrm{t})-\mathrm{R}(\mathrm{t})\right]
$$

where $R(t)=$ the photoadaptive variable of interest; $R_{w}(t)=$ the value which $R(t)$ would have if the phytoplankton were in balanced growth at the instantaneous $\mathrm{PFD}, \mathrm{I}(\mathrm{t}) ; \mathrm{k}=$ the rate constant for adaptation. Many photoadaptive variables can be characterized by first order rate constants 0.02 to 0.06 per hour (Falkowski 1980), which are of the same magnitude as the growth rate for many microalgae. It is well known that the rate 
constant for adaptation depends on the particular physiological process under consideration (Harris 1978). For example, the in vivo fluorescence properties of phytoplankton are characterized by faster response than chemical composition and the various parameters of the PI curve appear to adapt at different rates (Lewis et al. 1984a).

The empirical model (Eq. 1) has been applied in both theoretical (Falkowski \& Wirick 1981, Lewis et al. 1984a) and experimental (Falkowski 1980, Falkowski 1983, Lewis et al. 1984b) studies of phytoplankton photoadaptation in a wind-mixed euphotic zone. Justification for its use has been obtained in experimental studies on the responses of photoadaptive variables in unialgal cultures subjected to step changes in PFD (Rivkin et al. 1982a, Falkowski 1984, Lewis et al. 1984 a).

The theoretical link between observations of the chemical composition of microalgae in balanced growth and the changes in chemical composition during unbalanced growth has not, however, been previously established. Nor has a mechanistic derivation for the empirical photoadaptation model (Eq. 1) previously been given.

In this paper we present a time-dependent theory of photoadaptation of the microalgal cell using as an example the evolution of chla: $C$ during unbalanced growth.

\section{THEORY}

Consider the following equations which are proposed to describe the carbon and chlorophyll a dynamics of a microalgal cell population:

$$
\begin{aligned}
\mathrm{dC}(\mathrm{t}) / \mathrm{dt} & =\mathrm{p} C(\mathrm{t})-\mathrm{r}_{1} \mathrm{C}(\mathrm{t}) \\
\mathrm{dA}(\mathrm{t}) / \mathrm{dt} & =p \mathrm{f} C(\mathrm{t})-\mathrm{r}_{2} \mathrm{~A}(\mathrm{t})
\end{aligned}
$$

where $\mathrm{C}(\mathrm{t})=$ cell carbon concentration (mass of carbon per unit volume $\left.\left[\mathrm{M} / \mathrm{L}^{3}\right]\right) ; \mathrm{A}(\mathrm{t})=$ cell chlorophyll $a$ concentration (mass of carbon in chlorophyll a per unit volume $\left.\left[\mathrm{M} / \mathrm{L}^{3}\right]\right) ; \mathrm{p}=$ the rate constant for photosynthesis $\left(T^{-1}\right) ; r_{1}$ and $r_{2}=$ the rate constants for the degradation of, respectively, cell carbon and chlorophyll $a\left(\mathrm{~T}^{-1}\right)_{i} \mathrm{f}=$ the fraction of photosynthate which is directed into chlorophyll a synthesis (dimensionless). The differential equations (Eq. 2a, b) describe changes in the carbon and chlorophyll a concentrations resulting from imbalances between rates of synthesis and degradation. Synthesis and degradation are assumed to follow first order kinetics. Note that the concentrations of cell carbon and chlorophyll a are expressed in terms of carbon equivalents such that the fraction of photosynthate directed into chlorophyll a synthesis (f) is dimensionless. The equations for the microalgal population (Eq. 1 \& 2) are equally valid when recast in terms of individual microalgal cells. An advantage of Eq. 1 \& 2 is that they are directly applicable to observations made on cells in batch cultures.

For light-limited growth the photosynthesis rate (p) is assumed to be proportional to the PFD incident on the cell and the chla:C ratio:

$$
\mathrm{P}=a \gamma \mathrm{I}
$$

where $\mathrm{p}=$ the rate constant for photosynthesis $\left(\mathrm{s}^{-1}\right) ; \alpha$ $=$ initial slope of the PI curve (units of $[\mathrm{mg}$ total $\mathrm{C} / \mathrm{mg}$ $C$ in chla] $\left[\mathrm{m}^{2} /\right.$ umol photons $\left.]\right) ; \gamma=$ the chla: $C$ ratio (units of [ $\mathrm{mg} \mathrm{C}$ in chla] [mg C] ${ }^{-1}$ ); $\mathrm{I}=$ the photon flux density ( $\mu \mathrm{mol}$ photons $\mathrm{m}^{-2} \mathrm{~s}^{-1}$ ). Under the assumption that the degradation rate constarits $\left(r_{1}\right.$ and $\left.r_{2}\right)$ and the initial slope of the PI curve $(\alpha)$ are invariant, and that the proportion of photosynthate which is directed into chlorophyll a synthesis (f) changes instantaneously with changes in PFD, Eq. $2 a, 2 b$ and 3 can be used to solve for the time dependence of the C:chla ratio. Eq. 4 follows from Eq. 2a, $2 \mathrm{~b}$ and 3 upon noting the identity $\mathrm{d}(\mathrm{X} / \mathrm{Y}) / \mathrm{dt}=[\mathrm{Y}(\mathrm{dX} / \mathrm{dt})-\mathrm{X}(\mathrm{d} Y / \mathrm{dt})] / \mathrm{Y}^{2}$ and making the substitutions $X=A$ and $Y=C$ :

$$
d \theta(t) / d t=\alpha \operatorname{If}[(1 / f)-\theta(t)]-\theta(t)\left(r_{1}-r_{2}\right)
$$

where $\theta=C(t) / A(t)=1 / \gamma$ is the ratio of total carbon to carbon in chlorophyll $\alpha$. $r_{1}=r_{2}$, Eq. 4 reduces to:

$$
\mathrm{d} \theta(\mathrm{t}) / \mathrm{dt}=\alpha \operatorname{If}[(1 / \mathrm{f})-\theta(\mathrm{t})]
$$

Eq. 5 is the same as the empirical photoadaptation model (Eq. 1) given the following identifications: $\mathrm{k}=$ $(\alpha$ If $), R_{w}(t)=(1 / f), R(t)=\theta(t)$. The adaptation rate constant, $k$, is equal to the specific rate of synthesis of chlorophyll $a$ of fully adapted cells $(\mathrm{k}=\alpha$ If $=$ $\left.\left[\mathrm{A}(\mathrm{t})^{-1}\right][\mathrm{dA}(\mathrm{t}) / \mathrm{dt}]\right)$.

The dynamics of chla:C (i.e. $1 / \theta(t)=\gamma(t)$ ) derived from Eq. 2a, 2b and 3 will not yield such a simple equation. This can be shown as follows. Note the identity $d[1 / Z(t)] / d t=-Z(t)^{-2}[d Z(t) / d t]$ and substitute $\theta(\mathrm{t})=Z(\mathrm{t})$ to obtain Eq. 6 describing the time dependence of the chla:C ratio:

$$
\mathrm{d} \gamma(\mathrm{t}) / \mathrm{dt}=\mathrm{I}\left[\mathrm{f} \gamma(\mathrm{t})-\gamma(\mathrm{t})^{2}\right]
$$

Because of the $\gamma(t)^{2}$ term, this equation is non-linear.

The difference between the dynamics of chla:C (Eq. 5) and C:chla (Eq. 6) following a hypothetical step change in PFD is illustrated in Fig. 1 and 2. The ordinate is expressed in units of dimensionles time (i.e. the product of the photosynthesis rate and time). The trajectories followed by $\mathrm{C}$ :chla for reciprocal shifts in 


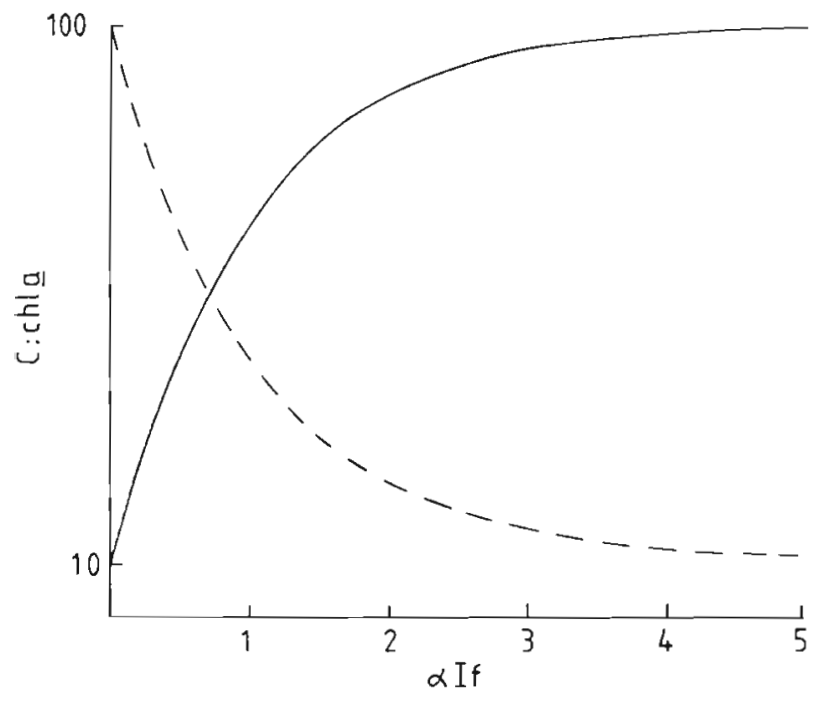

Fig. 1. Trajectory of the C:chla ratio predicted by Eq. 5 for hypothetical reciprocal shifts in PFD. The scale for the ordinate is dimensionless being set equal to the product of the adaptation rate constant ( $\alpha$ If) and time. Note the symmetry of the 2 trajectories

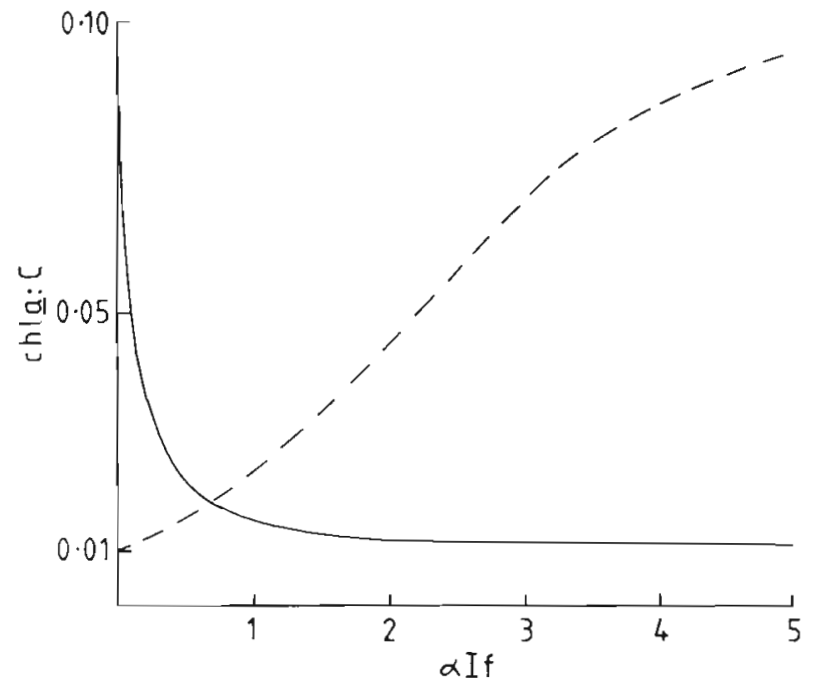

Fig. 2. Trajectory of the chla:C ratio predicted by Eq. 6 for the same hypothetical reciprocal shifts in PFD as are illustrated in Fig. 1. The scale for the ordinate is the same as in Fig. 1. Note that the magnitude of the asymmetry between the trajectories for the shift-up and shift-down experiments will depend on the range of values of the chla: $\mathrm{C}$ ratio which are produced by the PFD shift. The asymmetry is very pronounced for an order of magnitude change in the ratio but would be less marked for a 2 -fold change

PFD are symmetric, but the trajectories followed by chla:C are markedly asymmetric.

Now consider the application of Eq. $2 a, b$ to photoadaptation under conditions of light-saturated photosynthesis. In this case the photosynthesis rate (p) can be considered to be independent of both the PFD and the chla: $\mathrm{C}$ ratio, consistent with the observations of Myers (1970) for Chlorella vulgaris and Geider (1984) for Thalassiosira pseudonana. Under the assumption that the rate constant for light-saturated photosynthesis is constant, the coupled pair of differential equations describing changes in cell carbon and chlorophyll a content are:

$$
\begin{gathered}
d C(t) / d t=\left(P_{m}-r_{1}\right) C(t) \\
d A(t) / d t=P_{m} f C(t)-I_{2} A(t)
\end{gathered}
$$

where $\mathrm{P}_{\mathrm{m}}=$ the light-saturated photosynthesis rate constant $\left(\mathrm{T}^{-1}\right)$. Solving Eq. $7 \mathrm{a}$, b for chla: C yields Eq. 8:

$$
\mathrm{d} \gamma(\mathrm{t}) / \mathrm{dt}=\mathrm{P}_{\mathrm{m}}[\mathrm{f}-\gamma(\mathrm{t})]-\gamma(\mathrm{t})\left(\mathrm{r}_{2}-\mathrm{r}_{1}\right)
$$

If $r_{1}=r_{2}$, Eq. 8 reduces to:

$$
\mathrm{d} \gamma(\mathrm{t}) / \mathrm{dt}=\mathrm{P}_{\mathrm{m}}[\mathrm{f}-\gamma(\mathrm{t})]
$$

At saturating light levels chla: $\mathrm{C}$ follows adaptation kinetics (Eq. 9) consistent with the empirical model (Eq. 1) when the following indentities are noted; $\mathrm{k}=$ $\mathrm{P}_{\mathrm{m}}, \mathrm{R}_{\mathrm{w}}(\mathrm{t})=\mathrm{f}$, and $\mathrm{R}(\mathrm{t})=\gamma(\mathrm{t})$. By analogy with the derivation of Eq. 6 from Eq. 5, the dynamics of C:chla for growth within the light-saturated region of the PI curve can be derived from Eq. 9 to yield:

$$
\mathrm{d} \theta(\mathrm{t}) / \mathrm{dt}=\mathrm{P}_{\mathrm{m}}\left[\theta(\mathrm{t})-\mathrm{f} \theta(\mathrm{t})^{2}\right]
$$

The preceding analysis shows that even a simple model of carbon and chlorophyll a synthesis and degradation can lead to complex patterns of response of microalgal biochemical composition in fluctuating light. The non-linearity of the photosynthesis-light curve has significant effects on the rates of response of the chla: $\mathrm{C}$ and $\mathrm{C}$ :chla ratios following step shifts in PFD. The C:chla ratio is an example of a photoadaptive variable that is consistent with the empirical photoadaptation model (Eq. 1) for light-limited growth, whereas the chla: $\mathrm{C}$ ratio is consistent with the empirical model for light-saturated growth. The analysis shows that considerable care must be exercised in defining photoadaptive variables for use in empirical models if mechanistic interpretations are desired.

Explicit models of carbon flow through the microalgal cell, of which Eq. $2 \mathrm{a}$, b are simple examples, will further our understanding of the physiological aspects of photoadaptation kinetics in microalgae. The details made explicit in this approach may be unnecessary, and perhaps also computationally or analytically prohibitive, for some ecological applications. The physiological approach (Eq. 2a, b), however, does provide insight into choice of appropriate photoadaptive variables for use with the analytically tractable first order model (Eq. 1). 
The results presented here for the C:chla and chla:C ratios suggest that the photoadaptive variable appropriate for use in the empirical model is a ratio of biochemical properties of the algal cell in which the term in the denominator is proportional to the photosynthesis rate. In other words the denominator can be considered as the catalyst that limits the rate of photosynthesis. In the light-limited region of the PI curve, the photosynthesis rate is proportional to the chlorophyll a concentration because light harvesting limits photosynthesis. Chlorophyll $a$ is thus the catalyst limiting the rate of photosynthesis, and the photoadaptive variable which follows the first order adaptation model (Eq. 1) is the C:chla ratio. For light-saturated photosynthesis we have assumed that the photosynthesis rate is proportional to cell carbon, consistent with observations of Myers (1970) and Geider (1984). Although cell carbon is clearly not the catalyst which limits photosynthesis, the assumption that the carbonspecific, light-saturated photosynthesis rate is constant implies that the concentration of the rate-limiting catalyst is correlated with cell carbon. For light-saturated photosynthesis the photoadaptive variable which follows the kinetics described by Eq. 1 is the chla:C ratio.

In addition to identifying 'appropriate' photoadaptive variables, the mechanistic model (Eq. 2a, b) provides an interpretation of the first order rate constant for photoadaptation ( $\mathrm{k}$ in Eq. 1). If a photoadaptive variable is defined as a biochemical ratio in which the

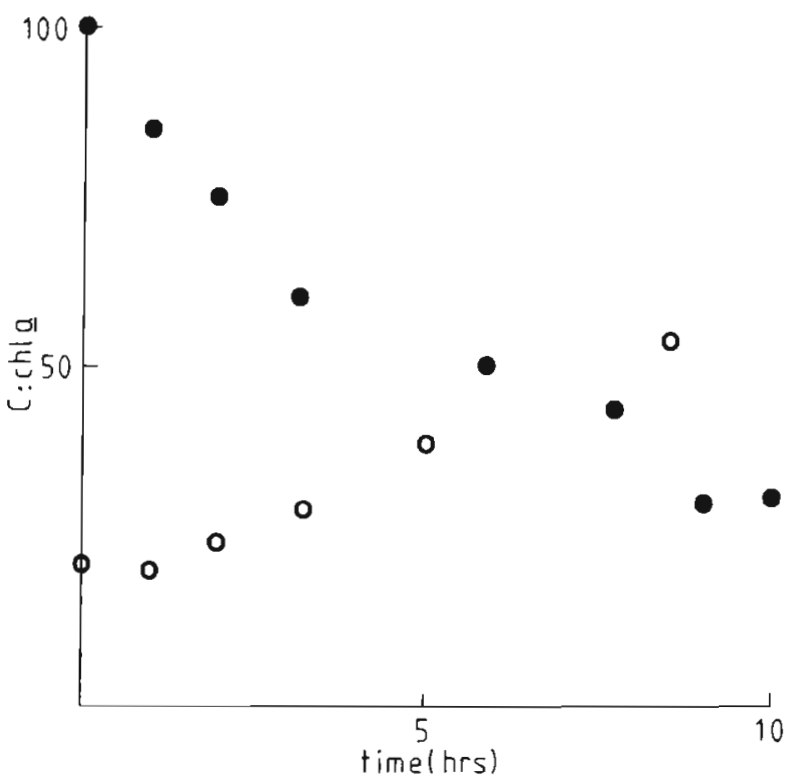

Fig. 3. Observations of changes in the C:chla ratio for Thalassiosira pseudonana cultures following reciprocal shifts in PFD between 100 and $2400 \mu \mathrm{mol}$ photons $\mathrm{m}^{-2} \mathrm{~s}^{-1}$. Open circles are observations for the shift-up in PFD. Closed circles are observations for the shift-down in PFD. Observations are taken from Lewis et al. (1984a)

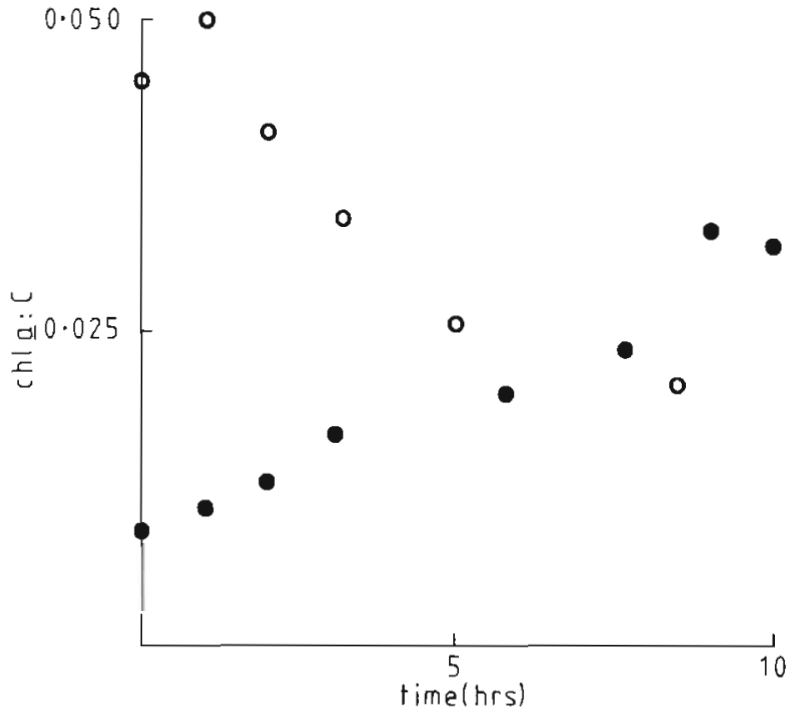

Fig. 4. Observations of changes in the chl a: C ratio for Thalassiosira pseudonana following reciprocal shifts in PFD between 100 and $2400 \mu \mathrm{mol}$ photons $\mathrm{m}^{-2} \mathrm{~s}^{-1}$. Open circles are observations for the shift-down in PFD for the same observations as are illustrated in Fig. 3

denominator is the catalyst limiting the rate of photosynthesis (or is directly correlated with the concentration of the rate-limiting catalyst), it follows that the adaptation rate constant $(\mathrm{k})$ will equal the balanced growth rate at the new light level. For the response of cell carbon and chlorophyll $a, k=P_{m}$ under lightsaturating conditions and $\mathrm{k}=\alpha$ If under light-limiting conditions.

\section{MODEL VERIFICATION}

The experimental study of the response of biochemical composition and photosynthesis rates of microalgae to step changes in PFD has been pursued for almost 25 yr (Steemann Nielsen et al. 1962, Sheridan 1972a, b, Beardall \& Morris 1976, Marra 1978, 1980, Prézelin \& Matlick 1980, Rivkin et al. 1984a). Most of these studies do not provide observations that can be used to test unambiguously the assumptions and predictions of the preceding theoretical analysis. One study (Lewis et al. 1984a), however, does provide observations which can be compared with the first order model (Eq. 5 and 9). We consider these observations next.

Observations of changes in C:chla in cultures of the marine diatom Thalassiosira pseudonana were interpreted by Lewis et al. (1984a) to indicate that chemical composition responded more slowly to a shift-up in PFD than to the reciprocal shift-down (Fig. 3). Reciprocal shifts in PFD were performed on cultures of T. pseudonana previously acclimated to light levels of 100 and $2400 \mu \mathrm{mol}$ photons $\mathrm{m}^{-2} \mathrm{~s}^{-1}$. Changes in C:chla 
for the reciprocal shifts are illustrated in Fig. 3 and changes in chla: $\mathrm{C}$ are illustrated in Fig. 4. Note the distinct asymmetry between the trajectories of both ratios for the reciprocal shifts in PFD.

The adaptation rate constants ( $k$ of Eq. 1) for the kinetics of C:chla during the first $10 \mathrm{~h}$ following a shift in PFD were determined from non-linear, least squares fits of the data of the integrated form of Eq. 1. The rate constants for shift-up and shift-down are respectively $0.05 \mathrm{~h}^{-1}$ and $0.2 \mathrm{~h}^{-1}$. The adaptation rate constants for shift-up and shift-down of chla: $\mathrm{C}$ are are respectively 0.14 and $0.083 \mathrm{~h}^{-1}$. The range of calculated adaptation rates $(k)$ for response of carbon and chlorophyll a varies by about a factor of 3 for the shift-up and 2 for the shiftdown depending on the choice of photoadaptive variable. Using $C$ :chla as a photoadaptive variable with Eq. 1 leads to the conclusion that photoadaptation following a shift-up in PFD occurs more slowly than adaptation to a shift-down in PFD; however, using chla: C as the photoadaptive variable leads to the opposite conclusion that adaptation following a shift-up occurs more rapidly than adaptation following a shiftdown in PFD. Rather than choosing between these 2 paradoxical interpretations based on fitting the observations to an empirical model, we can refer to the mechanistic model for insight into the underlying processes.

The high PFD (2400 $\left.\mu \mathrm{mol} \mathrm{m} \mathrm{m}^{-2} \mathrm{~s}^{-1}\right)$ is saturating for both growth and photosynthesis. The rate constant for the changes in the chla: $\mathrm{C}$ following the shift-up in light level should be $\mathrm{k}=\mathrm{P}_{\mathrm{m}}$ (Eq. 9), which, for Thalassiosira pseudonana growing at $100 \mu \mathrm{mol} \mathrm{m} \mathrm{m}^{-2} \mathrm{~s}^{-1}$, is $0.15 \mathrm{~h}^{-1}$. This value is similar to the light-saturated growth rate of $0.14 \mathrm{~h}^{-1}$ (M. Lewis unpubl. data). The adaptation rate constant ( $k$ of Eq. 1) calculated for this shift is $0.14 \mathrm{~h}^{-1}$, consistent with the maximum growth rate and light-saturated photosynthesis rate as predicted by the mechanistic model (Eq. 9). The low light level $\left(100 \mu \mathrm{mol} \mathrm{m} \mathrm{m}^{-2} \mathrm{~s}^{-1}\right)$ is within the linear, initial slope region of the PI curve for both the high-light and low-light acclimated cultures. As shown previously (Eq. 5), the adaptation rate constant for C:chla following a shift within the light-limited region of the PI curve should be $\mathrm{k}=(\alpha \mathrm{I})$, which for $\alpha=0.02(\mathrm{~g} \mathrm{C} / \mathrm{g}$ chla) $\left(\mathrm{m}^{2} / \mu \mathrm{mol}\right.$ photons), $\mathrm{I}=100 \mu \mathrm{mol} \mathrm{m} \mathrm{m}^{-2} \mathrm{~s}^{-1}$, and $\mathrm{f}=$ $0.05 \mathrm{~g}$ chla $\left(\mathrm{g} \mathrm{C}^{-1}\right.$, leads to $\mathrm{k}=0.1 \mathrm{~h}^{-1}$. This is only $1 / 2$ of the value of $\mathrm{k}=0.2 \mathrm{~h}^{-1}$ obtained from fitting Eq. 1 to the observed changes in C:chla. This inconsistency between observation and the mechanistic model (Eq. 5) may be due to a rapid utilization of energy reserves immediately following the shift-down in PFD. Consistent with this explanation is a reduction in total carbohydrate from 35 to $12 \%$ of the cell carbon within the first $2 \mathrm{~h}$ of the shift-down in PFD (calculated from Fig. 3 of Lewis et al. 1984a). We return to the potential role of energy reserves for adaptation kinetics in the discussion.

This reanalysis of the observations of Lewis et al. (1984a) for carbon and chlorophyll a dynamics is consistent with the predictions of the theoretical analysis. The interpretation of the observations in terms of the mechanistic model leads to the conciusion that the adaptation rates for the reciprocal shifts between 2400 and $100 \mathrm{~mol}$ photon $\mathrm{m}^{-1} \mathrm{~s}^{-1}$ will occur at approximately the same rate, but that the 'appropriate' variable for use with Eq. 1 is C:chla for the shift-down and chla: $\mathrm{C}$ for the shift-up. This conclusion would not have been obtained by simply fitting an arbitrarily chosen photoadaptive variable (i.e. either chla:carbon or carbon:chla) to the empirical model (Eq. 1).

\section{DISCUSSION}

The mechanistic theory of photoadaptation kinetics proposed in the preceding section consists of a pair of differential equations describing the mass balances of chlorophyll $a$ and carbon during microalgal growth. The mechanistic model (Eq. 2a, b) reduces to a previously described empirical model (Eq. 1) under certain conditions. It provides a theoretical basis for choosing 'appropriate' photoadaptive variables for use in the empirical model and insight into the physiological significance of the coefficients of Eq. 1 as follows.

(1) The photoadaptive variable for use in the empirical model is defined as the ratio of the concentrations of 2 biochemical components of a microalgal cell in which the denominator of the ratio is the rate-limiting catalyst for photosynthesis (or is proportional to the concentration of the rate-limiting catalyst).

(2) For a step change in PFD the rate constant ' $k$ ' will equal the balanced growth rate (sensu Eppley 1980) that would eventually be obtained under the new experimental conditions.

(3) The coefficient $R_{w}$ of Eq. 1 is equal to the ratio of the amounts of photosynthate directed into synthesis of the biochemical components making up the numerator and denominator of the ratio defining the photoadaptive variable.

Our theoretical treatment of adaptation does not consider the biochemical mechanisms that are responsible for adaptation. These physiological processes are taken into account by the specification of a coefficient which describes the fraction of photosynthate (f) directed into the synthesis of the biochemical compound of interest. In the differential equations used to model carbon and chlorophyll a dynamics (Eq. $2 \mathrm{a}, \mathrm{b})$ it was assumed that this coefficient (f) responds instantaneously following changes in PFD. Thus, although the photoresponse of biochemical composi- 
tion is observed on a time scale associated with the rate of photosynthesis, implicit in the model is a much shorter time scale (assumed to be infinitesimally small for our analysis) associated with changes in $\mathrm{f}$. Research into the mechanism that underlies photoadaptation should then be directed into an evaluation of the factors controlling the synthesis of key biochemical components of the photosynthetic apparatus. One such mechanism has been discussed by Falkowski (1980) for control of chlorophyll synthesis in microalgae.

It is possible, without going into a detailed consideration of algal biochemistry, to derive values for the coefficients of the photoadaptation models (Eq. 1, 5 \& 9) from an intracellular compartment model of microalgal growth (Shuter 1979). This model is based on the flow of energy and carbon through 4 intracellular compartments: the photosynthetic apparatus, the synthetic apparatus, the structural material, and the energy reserves. Under conditions of constant temperature and saturating nutrient availability, the model describes the distribution of carbon among the intracellular compartments and the growth rate as a function of PFD given the specification of 2 rate constants (i.e. a rate constant for photosynthesis, and a rate constant for the elaboration of the initial products of photosynthesis into new cells) and the energy costs of synthesis and maintenance. This model can be used to describe the dependencies of C:chla and growth rate on PFD for microalgae in balanced growth and thus provides a means for specifying the coefficient $f$ and the adaptation rate constant $\mathrm{k}$.

A consideration of the intracellular compartmental model of Shuter (1979) also highlights some of the limitations of the description of carbon and chlorophyll a dynamics by Eq. 2a, b. By considering only chlorophyll a and carbon dynamics, our analysis has been limited to a treatment of photosynthesis, implicitly neglecting the rate of elaboration of the products of photosynthesis into the complex macromolecules which make up the cell. One may suspect that this latter process will control the adaptation rate under some circumstances. Also neglected in our analysis is the role of an energy storage pool that could act as a buffer in temporary exposures to extremes of PFD, providing an energy reserve to maintain synthesis rates at low PFD and a means of collecting energy in excess of the demands for cell synthesis at high PFDs. The importance of this energy reserve is illustrated by the observation of Rivkin et al. (1982b) that a steady division rate can be maintained at the expense of stored carbon for 2 generations following a step-down in PFD in the dinoflagellate Gonyaulax polyhedra. In another paper, Rivkin et al. (1982a) note that changes in the cell chlorophyll a content of Pyrocystis noctiluca do not occur until $2 \mathrm{~d}$ following a shift-down in PFD after which time Eq. 1 adequately describes the photoresponse of cell chlorophyll. One area in which further theoretical work should proceed is in the extension of Shuter's (1979) model of balanced growth into a dynamic model applicable to the description of microalgal metabolism in a changing environment

One of the assumptions allowing the derivation of Eq. 5 and 9 from Eq. $2 a$, $b$ is that the degradation rate constants for carbon and chlorophyll $a\left(\mathrm{r}_{1}\right.$ and $\left.\mathrm{I}_{2}\right)$ have the same value. This assumption is required to make the derivation of the mass balances for carbon and chlorophyll a collapse to the empirical model. Carbon14 labelling experiments with microalgae in balanced growth indicate that total carbon and chlorophyll a label at the same rates (Redalje \& Laws 1981, Welschmeyer \& Lorenzen 1984) consistent with the assumption that $r_{1}=r_{2}$. Observations of rapid chlorophyll tumover (Grumbach et al. 1978, Riper et al. 1979), however, may indicate that this assumption is not universally valid.

Differences in degradation rate constants for chlorophyll $a$ and carbon will not significantly alter the kinetics of response of biochemical composition provided the synthesis rate constant ( $p$ ) is large relative to the degradation rate constants (see Eq. 4 \& 8). The linear first order models (Eq. 5 \& 9) and by implication the empirical model (Eq. 1) may adequately describe changes in chemical composition in rapidly growing cultures subjected to shifts in PFD (Falkowski 1984, Lewis et al, 1984a) because the synthesis rates are much larger than the degradation rate in these cultures. Under conditions in which the degradation rate constants are of the same magnitude as the synthesis rate constant, such as might be expected for extreme light- or nutrient-limitation, the empirical adaptation model (Eq. 1) may prove inappropriate. To the best of our knowledge this model has not been applied to such extreme situations.

One of the interesting conclusions of the theoretical analysis is that the adaptation rate parameter of the empirical model ( $\mathrm{k}$ in Eq. 1) is expected to be variable. It is not our intent to consider in detail the implications of variability in $\mathrm{k}_{\text {; }}$ however, we note that the adaptation rate will covary with the photosynthesis rate. Thus, photoadaptation rates of nutrient-sufficient microalgae will be faster at high light levels than at low light. The ecological implications are that phytoplankton in a mixed layer will become acclimated to light levels in excess of the mean PFD and that 'adaptation' will be faster during the day than at night.

The significance of the results presented here relates to the success of a simple mass balance in explaining major features of the adaptation kinetics of biochemical composition in microalgae. A coupled pair of first 
order differential equations is used to describe the rate of change of C:chla in microalgae. Other photoadaptive variables that can be expressed as the ratio of 2 chemical components should be able to be treated by a similar formulation. The model is not intended to apply to rapid changes in the fluorescence properties or photosynthesis rates (Kiefer 1973, Harris 1978) caused by changes in the biophysical or biochemical activity of a particular molecule or assemblage of molecules such as the photosynthetic unit. Neglect of variability in the activity of rate-limiting catalysts may be an important limitation of our treatment since both Lewis et al. (1984a) and Falkowski (1984) report that the initial slope of the PI curve can change following a shift in PFD. Our treatment also does not apply to diel periodicities in the value of a photoadaptive variable which result from cell cycle entrainment into a light: dark cycle or to circadian periodicities (Chisholm 1981). Rather, the model formulated in this paper applies to the synthesis and degradation of the various components which make up the algal cell. There is, however, no reason why the treatment cannot be extended to include a consideration of variable activity of the catalysts which limit the rate of photosynthesis.

Acknowledgements. This work was initiated as part of R. G.'s Ph. D. research in the Department of Oceanography, Dalhousie University where he was supported by Isaac Walton Killam and Dalhousie University Fellowships. R. Gis work in Prof. J. A. Raven's laboratory at The University of Dundee is supported by SERC. Comments and suggestions at various stages in the preparation of the manuscript were offered by C. J. Garrett, W. G. Harrison, M. Lewis, B. A. Osborne and R. E. H. Smith.

\section{LITERATURE CITED}

Bannister, T. T. (1979). Quantitative description of steadystate, nutrient-saturated algal growth including adaptation. Limnol. Oceanogr. 24: 76-96

Beardall, J., Morris, I. (1976). The concept of light intensity adaptation in marine phytoplankton: some experiments with Phaeodactylum tricornutum. Mar. Biol. 37: 377-387

Chisholm, S. W. (1981). Temporal pattern of cell division in unicellular algae. In: Platt, T. (ed.) Physiological bases of phytoplankton ecology. Can. Bull. Fish. Aquat. Sci. 201, p. 150-181

Collins, C. D., Boylen, C. W. (1982). Ecological consequences of long-term exposure of Anabaena variabilis (cyanophyceae) to shifts in environmental factors. Appl. environ. Microbiol. 44: 141-148

Cook, J. R. (1963). Adaptations in growth and division in Euglena effected by energy supply. J. Protozool. 10: $436-444$

Eppley, R. W. (1980). Estimating phytoplankton growth rates in oligotrophic oceans. In: Falkowski, P. G. (ed.) Primary productivity in the sea. Plenum Press, New York, p. 231-242

Falkowski, P. G. (1980). Light-shade adaptation and vertical mixing of marine phytoplankton: a comparative field study. J. mar. Res. 41: 215-237
Falkowski, P. G. (1984). Kinetics of adaptation to irradiance in Dunaliella tertiolecta. Photosynthetica 18: 62-68

Falkowski, P. G., Wirick, C. D. (1981). A simulation model of the effects of vertical mixing on primary productivity. Mar. Biol. 65: 69-75

Foy, R. H., Gibson, C. E. (1982). Photosynthetic characteristics of planktonic blue-green algae: changes in photosynthetic capacity and pigmentation of Oscillatoria redekei Van Goor under high and low light. Br. phycol. J. 17: 183-193

Geider, R. J. (1984). Light and nutrient effects on microalgal physiology. Ph. D. dissertation, Dalhousie University

Grumbach, K. H., Lichtenthaler, H. K., Erismann, K. H. (1978). Incorporation of ${ }^{14} \mathrm{CO}_{2}$ in photosynthetic pigments of Chlorella pyrenoidosa. Planta 140: 37-43

Harris, G. P. (1978). Photosynthesis, productivity and growth: the physiological ecology of phytoplankton. Arch. Hydrobiol. Beih. Ergeb. Limnol. 10: 1-69

Jones, R. I. (1978). Adaptations to fluctuating irradiance by natural phytoplankton communities. Limnol. Oceanogr. 23: 920-926

Kiefer, D. A. (1973). Chlorophyll a fluorescence in marine centric diatoms: responses of chloroplasts to light and nutrient stress. Mar. Biol. 23: 39-46

Kiefer, D. A., Mitchell, B. G. (1983). A simple, steady-state description of phytoplankton growth based on absorption cross-section and quantum efficiency. Limnol. Oceanogr. 28: $770-776$

Laws, E. A., Redalje, D. G., Karl, D. M., Chalup, M. S. (1983). A theoretical and experimental examination of the predictions of two recent models of phytoplankton growth. J. theor. Biol. 105: 469-491

Laws, E. A., Jones, D. R., Terry, K. L., Hirata, J. A. (1985). Modifications in recent models of phytoplankton growth: theoretical developments and experimental examination of predictions. J. theor. Biol. 114: 323-341

Lewis, M. R., Cullen, J. J., Platt, T. (1984a). Relationships between vertical mixing and photoadaptation of phytoplankton: similarity criteria. Mar Ecol. Prog. Ser. 15: 141-149

Lewis, M. R., Horne, E. P. W., Cullen, J. J, Oakey, N. S., Platt, T. (1984b). Turbulent motions may control phytoplankton photosynthesis in the upper ocean. Nature, Lond. 311: $49-50$

Liou, J. K., van Eybergen, G. C. (1982). Light adaptation and inhibition: processes important in modelling the growth of algae in drinking water basins. Wat. Res. 16: 765-773

Marra, J. (1978). Phytoplankton photosynthesis response to vertical motion in a mixed layer. Mar. Biol. 46: 203-208

Marra, J. (1980). Time course of light intensity adaptation in a marine diatom. Mar. Biol. Lett. 1: 175-183

Myers, J. (1970). Genetic and adaptive physiological characteristics observed in the chlorellas. In: Proc. IBP/PP technical meeting, Trebon, Prediction and measurement of photosynthetic production. Centre for Agricultural Publishing, Wageningen, p. 447-454

Parrot, L. M., Slater, J. H. (1980). The DNA, RNA and protein composition of the cyanobacterium Anacystis nidulans grown in light- and carbon dioxide-limited chemostats. Arch. Mikrobiol. 127: 53-58

Prézelin, B. B., Matlick, H. A. (1980). Time course of photoadaptation in the photosynthesis-irradiance relationship of a dinoflagellate exhibiting photosynthetic periodicity. Mar. Biol. 58: 85-96

Redalje, D. G., Laws, E. A. (1981). A new method for estimating phytoplankton growth rates and carbon biomass. Mar. Biol, 62: 73-79

Richardson, K., Beardall, J., Raven, J. A. (1983). Adaptation of 
unicellular algae to irradiance: an analysis of strategies. New Phytol 93: 157-191

Riper, D. M., Owens, T G., Falkowski, P. G. (1979). Chlorophyll turnover in Skeletonema costatum, a marine plankton diatom. Pl. Physiol., Lancaster 64: 49-54

Rivkin, R. B., Seliger, H. H., Swift, E., Biggley, W. H. (1982a). Light-shade adaptation by the oceanic dinoflagellates Pyrocystis noctiluca and $P$. fusiformis. Mar. Biol. 68: $181-191$

Rivkin, R. B., Voytek, M. A., Seliger, H. H. (1982b). Phytoplankton division rates in light-limited environments: two strategies. Science 215: 1123-1125

Sheridan, R. P. (1972a). Adaptation to quantum flux by the Emerson photosynthetic unit. Pl. Physiol. Lancaster 50: 355-359
Sheridan, R. P. (1972b). Kinetics of chlorophyll a and platoquinone a changes in response to light intensity. J. Phycol. 8: $166-169$

Shuter, B. (1979). A model of physiological adaptation in unicellular algae. J. theor. Biol. 78: 519-552

Smith, J. C., Platt, T., Harrison, W. G. (1983). Photoadaptation of carboxylating enzymes and photosynthesis during a spring bloom. Prog. Oceanogr. 12: 425-459

Steemann Nielsen, E., Hansen, V. Kr., Jorgensen, E. G. (1962). The adaptation to different light intensities in Chlorella vulgaris and the time dependence upon transfer to a new light intensity. Physiologia Pl. 15: 505-517

Welschmeyer, N. A., Lorenzen, C. J. (1984). Carbon-14 labelling of phytoplankton carbon and chlorophyll a: determination of specific growth rates. Limnol. Oceanogr. 29: $135-145$

This article was submitted to the editor; it was accepted for printing on January 24, 1986 